Acta Crystallographica Section E

Structure Reports

Online

ISSN 1600-5368

\section{Saccharin, redetermined at 120 K: a three- dimensional hydrogen-bonded framework}

James L. Wardell, ${ }^{a}$ John N. Low ${ }^{b}$ and Christopher Glidewell ${ }^{\mathrm{C} *}$

a Instituto de Química, Departamento de Química Inorgânica, Universidade Federal do Rio de Janeiro, 21945-970 Rio de Janeiro, RJ, Brazil, 'bepartment of Chemistry, University of Aberdeen, Meston Walk, Old Aberdeen AB24 3UE, Scotland, and ${ }^{\mathbf{c}}$ School of Chemistry, University of St Andrews, Fife KY16 9ST, Scotland

Correspondence e-mail: cg@st-andrews.ac.uk

\section{Key indicators}

Single-crystal X-ray study

$T=120 \mathrm{~K}$

Mean $\sigma(\mathrm{C}-\mathrm{C})=0.003 \AA$

$R$ factor $=0.039$

$w R$ factor $=0.092$

Data-to-parameter ratio $=15.7$

For details of how these key indicators were automatically derived from the article, see http://journals.iucr.org/e.
Molecules of the title compound, $\mathrm{C}_{7} \mathrm{H}_{5} \mathrm{NO}_{3} \mathrm{~S}$, are linked by paired $\mathrm{N}-\mathrm{H} \cdots \mathrm{O}=\mathrm{C}$ hydrogen bonds into $R_{2}^{2}(8)$ dimers and these dimers are linked into a three-dimensional framework structure by a combination of three independent $\mathrm{C}-\mathrm{H} \cdots \mathrm{O}$ hydrogen bonds.

\section{Comment}

The structure of saccharin, (I), was determined some years ago (Bart, 1968; Okaya, 1969) using diffraction data collected at ambient temperature, and accordingly the precision of some of the interatomic distances is fairly modest. While in one report (Bart, 1968) the precision on the bond angles is satisfactory, in the other (Okaya, 1969) no s.u. values were quoted for the interbond angles. The molecules were reported to form centrosymmetric dimers constructed from paired $\mathrm{N}-$ $\mathrm{H} \cdot \mathrm{O}=\mathrm{C}$ hydrogen bonds.<smiles>O=C1NS(=O)(=O)c2ccccc21</smiles>

(I)

We have now taken the opportunity to redetermine this structure using diffraction data collected at 120 (2) K; this has permitted refinement to a rather lower $R$ factor and has provided interatomic distances of significantly higher precision (Fig. 1 and Table 1). The cell dimensions and space group indicate that the same phase is present at $120 \mathrm{~K}$ as at ambient temperature.

The molecules are linked by a combination of $\mathrm{N}-\mathrm{H} \cdots \mathrm{O}$ and $\mathrm{C}-\mathrm{H}$. . O hydrogen bonds in which all three $\mathrm{O}$ atoms act as acceptors (Table 2). The $\mathrm{N}-\mathrm{H} \cdots \mathrm{O}$ hydrogen bond, which utilizes a carbonyl $\mathrm{O}$ atom as acceptor, generates a centrosymmetric $R_{2}^{2}$ (8) dimer (Fig. 2), exactly as reported previously; for the sake of convenience, the reference molecule has been selected so that this dimer lies across $\left(\frac{1}{2}, \frac{1}{2}, \frac{1}{2}\right)$. These dimers are linked into a single three-dimensional framework by three independent $\mathrm{C}-\mathrm{H} \cdots \mathrm{O}$ hydrogen bonds, each utilizing a different $\mathrm{O}$ atom as acceptor (Table 2). The hydrogen bond involving $\mathrm{C} 2$ as donor links the $R_{2}^{2}(8)$ dimer centred at $\left(\frac{1}{2}, \frac{1}{2}, \frac{1}{2}\right)$ to those centred at $\left(-\frac{1}{2}, 0,0\right),\left(-\frac{1}{2}, 1,0\right),\left(\frac{3}{2}, 0,1\right)$ and $\left(\frac{3}{2}, 1,1\right)$, thereby generating a $(-102)$ sheet. The hydrogen bond involving $\mathrm{C} 4$ as the donor links the $\left(\frac{1}{2}, \frac{1}{2}, \frac{1}{2}\right)$ dimer to those centred at $\left(\frac{1}{2},-1,0\right),\left(\frac{1}{2},-1,1\right),\left(\frac{1}{2}, 2,0\right)$ and $\left(\frac{1}{2}, 2,1\right)$, so forming a (100) sheet. This sheet is reinforced by the third, rather
Received 20 May 2005 Accepted 24 May 2005 Online 31 May 2005
(C) 2005 International Union of Crystallography Printed in Great Britain - all rights reserved 


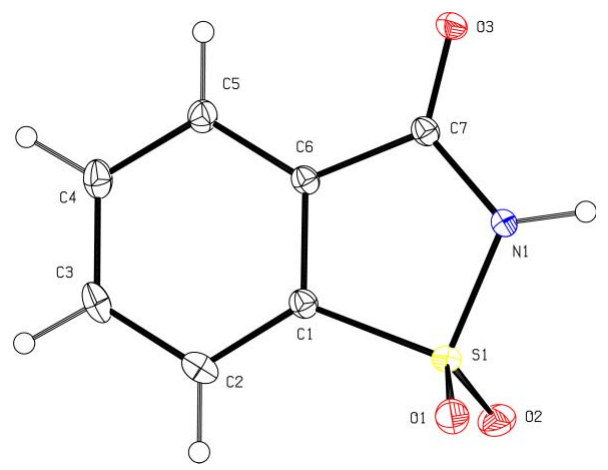

Figure 1

The molecule of (I), showing the atom-labelling scheme. Displacement ellipsoid are drawn at the $30 \%$ probability level.

weak, $\mathrm{C}-\mathrm{H}$... O hydrogen bond where C5 is the donor; this interaction links the $\left(\frac{1}{2}, \frac{1}{2}, \frac{1}{2}\right)$ dimer to those centred at $\left(\frac{1}{2}, 0,0\right)$, $\left(\frac{1}{2}, 1,0\right),\left(\frac{1}{2}, 0,1\right)$ and $\left(\frac{1}{2}, 1,1\right)$, so that the (100) sheet is of considerable complexity. The combination of the (100) and (102) sheets suffices to link all of the molecules into a single framework.

The original reports on the structure of (I) (Bart, 1968; Okaya, 1969) made no mention of the $\mathrm{C}-\mathrm{H} \cdots \mathrm{O}$ hydrogen bonds; at the time of those reports, the notion that such interactions could be of structural significance was not widely recognized and certainly not widely accepted.

\section{Experimental}

Crystals of compound (I) suitable for single-crystal X-ray diffraction were grown from an ethanol solution.

\section{Crystal data}

$\mathrm{C}_{7} \mathrm{H}_{5} \mathrm{NO}_{3} \mathrm{~S}$

$M_{r}=183.18$

Monoclinic, $P 2_{1} / c$

$a=9.4722(4) \mathrm{A}$

$b=6.9227(2) \AA$

$c=11.7322(3) \AA$

$\beta=103.203(3)^{\circ}$

$V=748.98(4) \AA^{3}$

$Z=4$

\section{Data collection}

Nonius KappaCCD diffractometer $\varphi$ and $\omega$ scans

Absorption correction: multi-scan

(SADABS; Sheldrick, 2003)

$T_{\min }=0.847, T_{\max }=0.958$

12303 measured reflections

1706 independent reflections

\section{Refinement}

Refinement on $F^{2}$

$R\left[F^{2}>2 \sigma\left(F^{2}\right)\right]=0.039$

$w R\left(F^{2}\right)=0.092$

$S=1.11$

1706 reflections

109 parameters

$\mathrm{H}$-atom parameters constrained

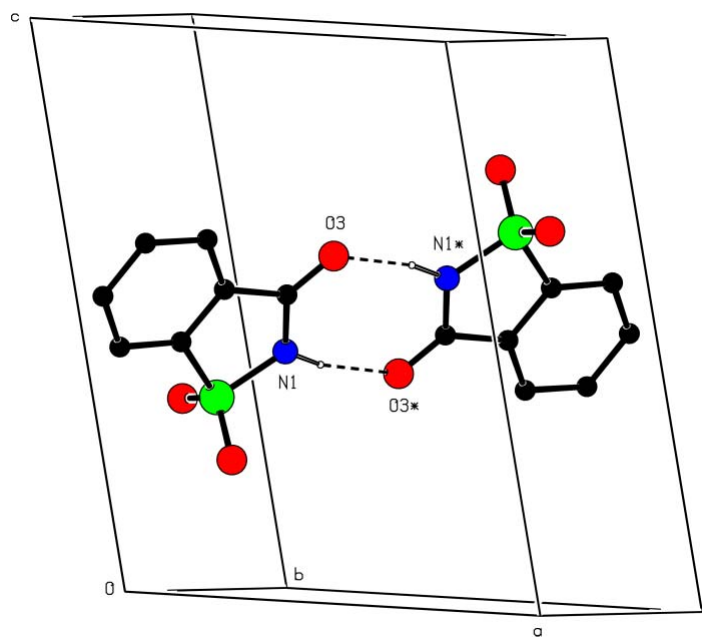

Figure 2

Part of the crystal structure of (I), showing the formation of an $R_{2}^{2}(8)$ dimer. For clarity, $\mathrm{H}$ atoms bonded to $\mathrm{C}$ atoms have been omitted. Hydrogen bonds are indicated by dashed lines. Atoms marked with an asterisk $(*)$ are at the symmetry position $(1-x, 1-y, 1-z)$.

Table 1

Selected geometric parameters $\left(\AA{ }^{\circ}\right)$.

\begin{tabular}{llll}
\hline S1-O1 & $1.4291(15)$ & $\mathrm{C} 1-\mathrm{C} 2$ & $1.387(3)$ \\
S1-O2 & $1.4323(15)$ & $\mathrm{C} 2-\mathrm{C} 3$ & $1.393(3)$ \\
S1-N1 & $1.6643(16)$ & $\mathrm{C} 3-\mathrm{C} 4$ & $1.390(3)$ \\
$\mathrm{S} 1-\mathrm{C} 1$ & $1.7560(19)$ & $\mathrm{C} 4-\mathrm{C} 5$ & $1.392(3)$ \\
$\mathrm{N} 1-\mathrm{C} 7$ & $1.374(2)$ & $\mathrm{C} 5-\mathrm{C} 6$ & $1.382(3)$ \\
$\mathrm{C} 7-\mathrm{O} 3$ & $1.223(2)$ & $\mathrm{C} 6-\mathrm{C} 1$ & $1.391(3)$ \\
$\mathrm{C} 7-\mathrm{C} 6$ & $1.481(3)$ & & \\
$\mathrm{O} 1-\mathrm{S} 1-\mathrm{O} 2$ & $117.37(9)$ & $\mathrm{S} 1-\mathrm{N} 1-\mathrm{C} 7$ & $115.65(13)$ \\
$\mathrm{O} 1-\mathrm{S} 1-\mathrm{N} 1$ & $110.37(9)$ & $\mathrm{O} 3-\mathrm{C} 7-\mathrm{N} 1$ & $124.53(17)$ \\
$\mathrm{O} 2-\mathrm{S} 1-\mathrm{N} 1$ & $109.48(9)$ & $\mathrm{O} 3-\mathrm{C} 7-\mathrm{C} 6$ & $126.12(17)$ \\
$\mathrm{O} 1-\mathrm{S} 1-\mathrm{C} 1$ & $111.71(9)$ & $\mathrm{N} 1-\mathrm{C} 7-\mathrm{C} 6$ & $109.34(16)$ \\
$\mathrm{O} 2-\mathrm{S} 1-\mathrm{C} 1$ & $112.68(9)$ & $\mathrm{S} 1-\mathrm{C} 1-\mathrm{C} 6$ & $110.01(14)$ \\
$\mathrm{N} 1-\mathrm{S} 1-\mathrm{C} 1$ & $92.41(8)$ & & \\
\hline
\end{tabular}

Table 2

Hydrogen-bond geometry $\left(\AA,^{\circ}\right)$.

\begin{tabular}{lllll}
\hline$D-\mathrm{H} \cdots A$ & $D-\mathrm{H}$ & $\mathrm{H} \cdots A$ & $D \cdots A$ & $D-\mathrm{H} \cdots A$ \\
\hline $\mathrm{N} 1-\mathrm{H} 1 \cdots \mathrm{O} 3$ & \\
$\mathrm{C} 2-\mathrm{H} 2 \cdots \mathrm{O} 2^{\text {ii }}$ & 0.95 & 1.86 & $2.786(2)$ & 167 \\
$\mathrm{C} 4-\mathrm{H} 4 \cdots \mathrm{O} 1^{\text {iii }}$ & 0.95 & 2.46 & $3.377(3)$ & 161 \\
$\mathrm{C} 5-\mathrm{H} 5 \cdots 3^{\text {iv }}$ & 0.95 & 2.55 & $3.375(2)$ & 145 \\
\end{tabular}

1490 reflections with $I>2 \sigma(I)$

$R_{\text {int }}=0.052$

$\theta_{\max }=27.5^{\circ}$

$h=-11 \rightarrow 12$

$k=-8 \rightarrow 9$

$l=-14 \rightarrow 15$

Symmetry codes: (i) $-x+1,-y+1,-z+1$; (ii) $-x, y-\frac{1}{2},-z+\frac{1}{2}$; (iii) $x,-y-\frac{1}{2}, z+\frac{1}{2}$; (iv) $-x+1, y-\frac{1}{2},-z+\frac{3}{2}$.

All $\mathrm{H}$ atoms were located in difference maps and then treated as riding atoms with $\mathrm{C}-\mathrm{H}=0.95 \AA$ and $\mathrm{N}-\mathrm{H}=0.95 \AA$, and with $U_{\text {iso }}(\mathrm{H})=1.2 U_{\text {eq }}(\mathrm{C}, \mathrm{N})$.

Data collection: COLLECT (Hooft, 1999); cell refinement: DENZO (Otwinowski \& Minor, 1997) and COLLECT; data reduction: DENZO and COLLECT; program(s) used to solve structure: WinGX (Farrugia, 1999) and SIR92 (Altomare et al., 1993); program(s) used to refine structure: OSCAIL (McArdle, 2003) and SHELXL97 (Sheldrick, 1997); molecular graphics: PLATON (Spek, 2003); software used to prepare material for publication: SHELXL97 and PRPKAPPA (Ferguson, 1999). 
X-ray data were collected at the EPSRC X-ray Crystallographic Service, University of Southampton, England; the authors thank the staff for all their help and advice. JLW thanks CNPq and FAPERJ for financial support.

\section{References}

Altomare, A., Cascarano, G., Giacovazzo, C. \& Guagliardi, A. (1993). J. Appl. Cryst. 26, 343-350.

Bart, J. C. J. (1968). J. Chem. Soc. B, pp. 376-382.
Farrugia, L. J. (1999). J. Appl. Cryst. 32, 837-838.

Ferguson, G. (1999). PRPKAPPA. University of Guelph, Canada.

Hooft, R. W. W. (1999). COLLECT. Nonius BV, Delft, The Netherlands.

McArdle, P. (2003). OSCAIL for Windows. Version 10. Crystallography

Centre, Chemistry Department, NUI Galway, Ireland.

Okaya, Y. (1969). Acta Cryst. B25, 2257-2263.

Otwinowski, Z. \& Minor, W. (1997). Methods in Enzymology, Vol. 276 Macromolecular Crystallography, Part A, edited by C. W. Carter Jr \& R. M. Sweet, pp. 307-326. New York: Academic Press.

Sheldrick, G. M. (1997). SHELXL97. University of Göttingen, Germany.

Sheldrick, G. M. (2003). SADABS. Version 2.10. University of Göttingen, Germany.

Spek, A. L. (2003). J. Appl. Cryst. 36, 7-13. 


\section{supporting information}

Acta Cryst. (2005). E61, o1944-o1946 [https://doi.org/10.1107/S1600536805016600]

Saccharin, redetermined at $120 \mathrm{~K}$ : a three-dimensional hydrogen-bonded framework

James L. Wardell, John N. Low and Christopher Glidewell

Saccharin

Crystal data

$\mathrm{C}_{7} \mathrm{H}_{5} \mathrm{NO}_{3} \mathrm{~S}$

$M_{r}=183.18$

Monoclinic, $P 2_{1} / c$

Hall symbol: -P 2ybc

$a=9.4722(4) \AA$

$b=6.9227(2) \AA$

$c=11.7322(3) \AA$

$\beta=103.203(3)^{\circ}$

$V=748.98(4) \AA^{3}$

$Z=4$

$F(000)=376$

$D_{\mathrm{x}}=1.624 \mathrm{Mg} \mathrm{m}^{-3}$

Mo $K \alpha$ radiation, $\lambda=0.71073 \AA$

Cell parameters from 1706 reflections

$\theta=3.6-27.5^{\circ}$

$\mu=0.39 \mathrm{~mm}^{-1}$

$T=120 \mathrm{~K}$

Lath, colourless

$0.44 \times 0.16 \times 0.11 \mathrm{~mm}$

Data collection

Nonius KappaCCD

diffractometer

Radiation source: Bruker-Nonius FR91 rotating anode

Graphite monochromator

Detector resolution: 9.091 pixels $\mathrm{mm}^{-1}$

$\varphi$ and $\omega$ scans

Absorption correction: multi-scan

(SADABS; Sheldrick, 2003)

$T_{\min }=0.847, T_{\max }=0.958$

12303 measured reflections

1706 independent reflections

1490 reflections with $I>2 \sigma(I)$

$R_{\text {int }}=0.052$

$\theta_{\max }=27.5^{\circ}, \theta_{\min }=3.6^{\circ}$

$h=-11 \rightarrow 12$

$k=-8 \rightarrow 9$

$l=-14 \rightarrow 15$

Refinement

Refinement on $F^{2}$

Least-squares matrix: full

$R\left[F^{2}>2 \sigma\left(F^{2}\right)\right]=0.039$

$w R\left(F^{2}\right)=0.092$

$S=1.11$

1706 reflections

109 parameters

0 restraints

Primary atom site location: structure-invariant

direct methods

Secondary atom site location: difference Fourier map

Hydrogen site location: inferred from neighbouring sites

$\mathrm{H}$-atom parameters constrained

$w=1 /\left[\sigma^{2}\left(F_{\mathrm{o}}^{2}\right)+(0.0203 P)^{2}+0.8656 P\right]$

where $P=\left(F_{\mathrm{o}}^{2}+2 F_{\mathrm{c}}^{2}\right) / 3$

$(\Delta / \sigma)_{\max }<0.001$

$\Delta \rho_{\max }=0.42$ e $\AA^{-3}$

$\Delta \rho_{\min }=-0.63{\mathrm{e} \AA^{-3}}^{-3}$

Fractional atomic coordinates and isotropic or equivalent isotropic displacement parameters $\left(\AA^{2}\right)$

\begin{tabular}{lllll}
\hline & $x$ & $y$ & $z$ & $U_{\text {iso }} * / U_{\text {eq }}$ \\
\hline $\mathrm{S} 1$ & $0.21067(5)$ & $0.22909(7)$ & $0.34594(4)$ & $0.01955(15)$
\end{tabular}




$\begin{array}{lllll}\text { O1 } & 0.24538(17) & 0.1705(2) & 0.23876(12) & 0.0271(3) \\ \text { O2 } & 0.08217(15) & 0.3423(2) & 0.33788(13) & 0.0287(4) \\ \text { O3 } & 0.52054(15) & 0.3079(2) & 0.60463(11) & 0.0216(3) \\ \text { N1 } & 0.35108(18) & 0.3420(2) & 0.43105(14) & 0.0207(4) \\ \text { C1 } & 0.2223(2) & 0.0356(3) & 0.44441(16) & 0.0184(4) \\ \text { C2 } & 0.1361(2) & -0.1284(3) & 0.43285(18) & 0.0256(4) \\ \text { C3 } & 0.1685(2) & -0.2627(3) & 0.52349(19) & 0.0271(5) \\ \text { C4 } & 0.2818(2) & -0.2340(3) & 0.62062(18) & 0.0256(4) \\ \text { C5 } & 0.3685(2) & -0.0699(3) & 0.62963(17) & 0.0218(4) \\ \text { C6 } & 0.3373(2) & 0.0646(3) & 0.54037(15) & 0.0170(4) \\ \text { C7 } & 0.4147(2) & 0.2483(3) & 0.53308(16) & 0.0177(4) \\ \text { H1 } & 0.3838 & 0.4622 & 0.4087 & 0.025^{*} \\ \text { H2 } & 0.0588 & -0.1481 & 0.3664 & 0.031^{*} \\ \text { H3 } & 0.1117 & -0.3767 & 0.5188 & 0.033^{*} \\ \text { H4 } & 0.3004 & -0.3275 & 0.6815 & 0.031^{*} \\ \text { H5 } & 0.4470 & -0.0508 & 0.6953 & 0.026^{*}\end{array}$

Atomic displacement parameters $\left(\AA^{2}\right)$

\begin{tabular}{lllllll}
\hline & $U^{11}$ & $U^{22}$ & $U^{33}$ & $U^{12}$ & $U^{13}$ & $U^{23}$ \\
\hline S1 & $0.0205(3)$ & $0.0188(3)$ & $0.0173(2)$ & $-0.00189(18)$ & $0.00002(17)$ & $-0.00062(17)$ \\
O1 & $0.0372(9)$ & $0.0252(8)$ & $0.0189(7)$ & $-0.0036(6)$ & $0.0067(6)$ & $-0.0018(6)$ \\
O2 & $0.0221(8)$ & $0.0307(8)$ & $0.0303(8)$ & $0.0049(6)$ & $-0.0002(6)$ & $0.0014(6)$ \\
O3 & $0.0239(7)$ & $0.0222(7)$ & $0.0169(6)$ & $-0.0054(6)$ & $0.0008(5)$ & $-0.0016(5)$ \\
N1 & $0.0235(9)$ & $0.0165(8)$ & $0.0195(8)$ & $-0.0048(6)$ & $-0.0003(6)$ & $0.0015(6)$ \\
C1 & $0.0204(9)$ & $0.0176(9)$ & $0.0176(9)$ & $-0.0012(7)$ & $0.0053(7)$ & $-0.0021(7)$ \\
C2 & $0.0225(10)$ & $0.0275(11)$ & $0.0264(10)$ & $-0.0062(9)$ & $0.0048(8)$ & $-0.0070(9)$ \\
C3 & $0.0307(11)$ & $0.0191(10)$ & $0.0349(11)$ & $-0.0085(8)$ & $0.0146(9)$ & $-0.0033(8)$ \\
C4 & $0.0344(12)$ & $0.0202(10)$ & $0.0253(10)$ & $-0.0029(8)$ & $0.0130(9)$ & $0.0015(8)$ \\
C5 & $0.0279(10)$ & $0.0196(9)$ & $0.0186(9)$ & $-0.0012(8)$ & $0.0067(8)$ & $0.0001(7)$ \\
C6 & $0.0197(9)$ & $0.0161(9)$ & $0.0159(8)$ & $-0.0010(7)$ & $0.0056(7)$ & $-0.0034(7)$ \\
C7 & $0.0197(9)$ & $0.0168(9)$ & $0.0174(9)$ & $-0.0017(7)$ & $0.0057(7)$ & $-0.0027(7)$ \\
& & & & & &
\end{tabular}

Geometric parameters $(\AA, \stackrel{o}{)})$

\begin{tabular}{llll}
\hline $\mathrm{S} 1-\mathrm{O} 1$ & $1.4291(15)$ & $\mathrm{C} 2-\mathrm{C} 3$ & $1.393(3)$ \\
$\mathrm{S} 1-\mathrm{O} 2$ & $1.4323(15)$ & $\mathrm{C} 2-\mathrm{H} 2$ & 0.95 \\
$\mathrm{~S} 1-\mathrm{N} 1$ & $1.6643(16)$ & $\mathrm{C} 3-\mathrm{C} 4$ & $1.390(3)$ \\
$\mathrm{S} 1-\mathrm{C} 1$ & $1.7560(19)$ & $\mathrm{C} 3-\mathrm{H} 3$ & 0.95 \\
$\mathrm{~N} 1-\mathrm{C} 7$ & $1.374(2)$ & $\mathrm{C} 4-\mathrm{C} 5$ & $1.392(3)$ \\
$\mathrm{N} 1-\mathrm{H} 1$ & 0.9462 & $\mathrm{C} 4-\mathrm{H} 4$ & 0.95 \\
$\mathrm{C} 7-\mathrm{O} 3$ & $1.223(2)$ & $\mathrm{C} 5-\mathrm{C} 6$ & $1.382(3)$ \\
$\mathrm{C} 7-\mathrm{C} 6$ & $1.481(3)$ & $\mathrm{C} 5-\mathrm{H} 5$ & 0.95 \\
$\mathrm{C} 1-\mathrm{C} 2$ & $1.387(3)$ & $\mathrm{C} 6-\mathrm{C} 1$ & $1.391(3)$ \\
& & & $116.72(18)$ \\
$\mathrm{O} 1-\mathrm{S} 1-\mathrm{O} 2$ & $117.37(9)$ & $\mathrm{C} 1-\mathrm{C} 2-\mathrm{C} 3$ & 121.6 \\
$\mathrm{O} 1-\mathrm{S} 1-\mathrm{N} 1$ & $110.37(9)$ & $\mathrm{C} 1-\mathrm{C} 2-\mathrm{H} 2$ & 121.6 \\
$\mathrm{O} 2-\mathrm{S} 1-\mathrm{N} 1$ & $109.48(9)$ & $\mathrm{C} 3-\mathrm{C} 2-\mathrm{H} 2$ &
\end{tabular}




\begin{tabular}{|c|c|c|c|}
\hline $\mathrm{O} 1-\mathrm{S} 1-\mathrm{C} 1$ & $111.71(9)$ & $\mathrm{C} 4-\mathrm{C} 3-\mathrm{C} 2$ & $121.65(19)$ \\
\hline $\mathrm{O} 2-\mathrm{S} 1-\mathrm{C} 1$ & $112.68(9)$ & $\mathrm{C} 4-\mathrm{C} 3-\mathrm{H} 3$ & 119.2 \\
\hline $\mathrm{N} 1-\mathrm{S} 1-\mathrm{C} 1$ & $92.41(8)$ & $\mathrm{C} 2-\mathrm{C} 3-\mathrm{H} 3$ & 119.2 \\
\hline $\mathrm{S} 1-\mathrm{N} 1-\mathrm{C} 7$ & $115.65(13)$ & $\mathrm{C} 3-\mathrm{C} 4-\mathrm{C} 5$ & $120.71(19)$ \\
\hline $\mathrm{C} 7-\mathrm{N} 1-\mathrm{H} 1$ & 123.4 & $\mathrm{C} 3-\mathrm{C} 4-\mathrm{H} 4$ & 119.6 \\
\hline $\mathrm{S} 1-\mathrm{N} 1-\mathrm{H} 1$ & 120.9 & $\mathrm{C} 5-\mathrm{C} 4-\mathrm{H} 4$ & 119.6 \\
\hline $\mathrm{O} 3-\mathrm{C} 7-\mathrm{N} 1$ & $124.53(17)$ & $\mathrm{C} 6-\mathrm{C} 5-\mathrm{C} 4$ & $118.18(18)$ \\
\hline $\mathrm{O} 3-\mathrm{C} 7-\mathrm{C} 6$ & $126.12(17)$ & $\mathrm{C} 6-\mathrm{C} 5-\mathrm{H} 5$ & 120.9 \\
\hline $\mathrm{N} 1-\mathrm{C} 7-\mathrm{C} 6$ & $109.34(16)$ & $\mathrm{C} 4-\mathrm{C} 5-\mathrm{H} 5$ & 120.9 \\
\hline $\mathrm{C} 2-\mathrm{C} 1-\mathrm{C} 6$ & $122.17(18)$ & $\mathrm{C} 5-\mathrm{C} 6-\mathrm{C} 1$ & $120.57(18)$ \\
\hline $\mathrm{C} 2-\mathrm{C} 1-\mathrm{S} 1$ & $127.80(15)$ & $\mathrm{C} 5-\mathrm{C} 6-\mathrm{C} 7$ & $126.84(17)$ \\
\hline $\mathrm{S} 1-\mathrm{C} 1-\mathrm{C} 6$ & $110.01(14)$ & $\mathrm{C} 1-\mathrm{C} 6-\mathrm{C} 7$ & $112.59(16)$ \\
\hline $\mathrm{O} 1-\mathrm{S} 1-\mathrm{N} 1-\mathrm{C} 7$ & $-113.64(15)$ & $\mathrm{C} 1-\mathrm{C} 2-\mathrm{C} 3-\mathrm{C} 4$ & $-0.1(3)$ \\
\hline $\mathrm{O} 2-\mathrm{S} 1-\mathrm{N} 1-\mathrm{C} 7$ & $115.70(15)$ & $\mathrm{C} 2-\mathrm{C} 3-\mathrm{C} 4-\mathrm{C} 5$ & $-0.9(3)$ \\
\hline $\mathrm{C} 1-\mathrm{S} 1-\mathrm{N} 1-\mathrm{C} 7$ & $0.60(16)$ & $\mathrm{C} 3-\mathrm{C} 4-\mathrm{C} 5-\mathrm{C} 6$ & $1.0(3)$ \\
\hline $\mathrm{S} 1-\mathrm{N} 1-\mathrm{C} 7-\mathrm{O} 3$ & $178.42(15)$ & $\mathrm{C} 4-\mathrm{C} 5-\mathrm{C} 6-\mathrm{C} 1$ & $-0.1(3)$ \\
\hline $\mathrm{S} 1-\mathrm{N} 1-\mathrm{C} 7-\mathrm{C} 6$ & $-0.3(2)$ & $\mathrm{C} 4-\mathrm{C} 5-\mathrm{C} 6-\mathrm{C} 7$ & $179.60(18)$ \\
\hline $\mathrm{O} 1-\mathrm{S} 1-\mathrm{C} 1-\mathrm{C} 2$ & $-66.2(2)$ & $\mathrm{C} 2-\mathrm{C} 1-\mathrm{C} 6-\mathrm{C} 5$ & $-1.0(3)$ \\
\hline $\mathrm{O} 2-\mathrm{S} 1-\mathrm{C} 1-\mathrm{C} 2$ & $68.4(2)$ & $\mathrm{S} 1-\mathrm{C} 1-\mathrm{C} 6-\mathrm{C} 5$ & $-179.60(15)$ \\
\hline $\mathrm{N} 1-\mathrm{S} 1-\mathrm{C} 1-\mathrm{C} 2$ & $-179.26(19)$ & $\mathrm{C} 2-\mathrm{C} 1-\mathrm{C} 6-\mathrm{C} 7$ & $179.32(18)$ \\
\hline $\mathrm{O} 1-\mathrm{S} 1-\mathrm{C} 1-\mathrm{C} 6$ & $112.34(14)$ & $\mathrm{S} 1-\mathrm{C} 1-\mathrm{C} 6-\mathrm{C} 7$ & $0.7(2)$ \\
\hline $\mathrm{O} 2-\mathrm{S} 1-\mathrm{C} 1-\mathrm{C} 6$ & $-113.01(14)$ & $\mathrm{O} 3-\mathrm{C} 7-\mathrm{C} 6-\mathrm{C} 5$ & $1.3(3)$ \\
\hline $\mathrm{N} 1-\mathrm{S} 1-\mathrm{C} 1-\mathrm{C} 6$ & $-0.72(15)$ & $\mathrm{N} 1-\mathrm{C} 7-\mathrm{C} 6-\mathrm{C} 5$ & $-179.96(18)$ \\
\hline $\mathrm{C} 6-\mathrm{C} 1-\mathrm{C} 2-\mathrm{C} 3$ & $1.1(3)$ & $\mathrm{O} 3-\mathrm{C} 7-\mathrm{C} 6-\mathrm{C} 1$ & $-178.97(19)$ \\
\hline $\mathrm{S} 1-\mathrm{C} 1-\mathrm{C} 2-\mathrm{C} 3$ & $179.43(16)$ & $\mathrm{N} 1-\mathrm{C} 7-\mathrm{C} 6-\mathrm{C} 1$ & $-0.3(2)$ \\
\hline
\end{tabular}

Hydrogen-bond geometry $\left(\AA,{ }^{\circ}\right)$

\begin{tabular}{lllll}
\hline$D-\mathrm{H} \cdots A$ & $D-\mathrm{H}$ & $\mathrm{H} \cdots A$ & $D \cdots A$ & $D-\mathrm{H}^{\cdots} A$ \\
\hline $\mathrm{N} 1-\mathrm{H} 1 \cdots \mathrm{O} 3^{\mathrm{i}}$ & 0.95 & 1.86 & $2.786(2)$ & 167 \\
$\mathrm{C} 2-\mathrm{H} 2 \cdots \mathrm{O} 2^{\mathrm{ii}}$ & 0.95 & 2.46 & $3.377(3)$ & 161 \\
$\mathrm{C} 4-\mathrm{H} 4 \cdots \mathrm{O} 1^{\mathrm{iii}}$ & 0.95 & 2.55 & $3.375(2)$ & 145 \\
$\mathrm{C} 5-\mathrm{H} 5 \cdots 3^{\text {iv }}$ & 0.95 & 2.50 & $3.169(2)$ & 128 \\
\hline
\end{tabular}

Symmetry codes: (i) $-x+1,-y+1,-z+1$; (ii) $-x, y-1 / 2,-z+1 / 2$; (iii) $x,-y-1 / 2, z+1 / 2$; (iv) $-x+1, y-1 / 2,-z+3 / 2$. 